\title{
Redshift of southern radio galaxies ${ }^{\star}$
}

\author{
R. Scarpa ${ }^{1, \star \star}$, R. Falomo ${ }^{2}$ and J.E. Pesce ${ }^{3}$ \\ 1 Dipartimento di Astronomia Università di Padova, Via Osservatorio 5, 35122 Padova, Italy \\ E-mail: riccardo@astrpd.pd.astro.it \\ 2 Osservatorio Astronomico di Padova, Via Osservatorio 5, 35122 Padova, Italy \\ E-mail: falomo@astrpd.pd.astro.it \\ 3 Space Telescope Science Institute 3700 San Martin Drive, Baltimore, MD 21218, U.S.A. \\ E-mail pesce@stsci.edu
}

Received September 25; accepted September 27, 1995

\begin{abstract}
We report optical spectroscopy and redshift determination for 22 radio galaxies, extracted from the Ekers et al. (1989) compilation. Selected sources, which have both Fanaroff \& Riley type I and II radio morphology and are classified as elliptical or S0, have a typical early-type spectrum with strong absorption lines as generally observed in non-radio elliptical galaxies. Only in two cases, 0344-345 and 1521-300, moderately strong emission lines are observed.
\end{abstract}

Key words: galaxies: distances and redshift

\section{Introduction}

We are currently studying a large sample of radio galaxies in the southern hemisphere, both of Fanaroff \& Riley class I and II (FR) (Fanaroff \& Riley 1974). The sample is drawn from the lists of Wall \& Peacock (1985) and Ekers et al. (1989) selecting objects with optical counterpart classified as elliptic or S0, in the distance range $0.01<z<0.12$, in order to study their optical morphological and photometrical properties (Fasano et al. 1996) and the Megaparsec-scale environments (Pesce et al. 1996). The results from these studies will also be used to compare the radio galaxy optical properties with those of the BL Lacertae host galaxies. This is useful for testing the hypothesis that BL Lacs are radio galaxies with a relativistic jet closely aligned toward the observer line of sight (see e.g. Urry \& Padovani 1995 and references therein).

Because complete redshift information was not available for the Ekers et al. subsample (for the Wall \& Peacock list see Tadhunter et al. 1993), we undertook optical spectroscopic observations to obtain the missing values.

Send offprint requests to: R. Scarpa

${ }^{\star}$ Based on observations collected at the European Southern Observatory, La Silla, Chile

${ }^{\star \star}$ Present address: Space Telescope Science Institute 3700 San Martin Drive, Baltimore, MD 21218, U.S.A.
Table 1. Redshift of radio galaxies

\begin{tabular}{cccccc}
\hline \hline & & & & \\
Object & $\alpha \mathbf{( 1 9 5 0 )}$ & $\delta(\mathbf{1 9 5 0})$ & $m_{V}$ & redshift \\
\hline $0005-199$ & 000543 & -195630 & 16.8 & $0.1216 \pm 0.0005$ \\
$0013-316$ & 001345 & -314009 & 16.8 & $0.1071 \pm 0.0008$ \\
$0155-212$ & 015532 & -211652 & 17.8 & $0.1597 \pm 0.0004$ \\
$0229-208$ & 022918 & -205335 & 16.0 & $0.0895 \pm 0.0006$ \\
$0247-207$ & 024717 & -204256 & 15.4 & $0.0864 \pm 0.0007$ \\
$0257-398$ & 025732 & -395230 & 15.3 & $0.0661 \pm 0.0004$ \\
$0312-343$ & 031233 & -341846 & 15.6 & $0.0669 \pm 0.0004$ \\
$0344-345$ & 034435 & -343202 & 16.3 & $0.0535 \pm 0.0004$ \\
$0452-190$ & 045206 & -190017 & 14.8 & $0.0389 \pm 0.0002$ \\
$0533-377$ & 053340 & -374504 & 16.7 & $0.0964 \pm 0.0008$ \\
$0712-349$ & 071207 & -345612 & 15.9 & $0.0446 \pm 0.0003$ \\
$0940-304$ & 094012 & -303027 & 14.5 & $0.0385 \pm 0.0003$ \\
$1002-320$ & 100226 & -320207 & 17.4 & $0.0887 \pm 0.0008$ \\
$1043-290$ & 104348 & -290521 & 15.4 & $0.0598 \pm 0.0003$ \\
$1257-253$ & 125707 & -252329 & 16.0 & $0.0648 \pm 0.0006$ \\
$1354-251$ & 135427 & -250846 & 15.4 & $0.0379 \pm 0.0002$ \\
$1521-300$ & 152129 & -300146 & 15.3 & $0.0196 \pm 0.0004$ \\
$1929-397$ & 192959 & -394706 & 15.5 & $0.0744 \pm 0.0004$ \\
$2209-255$ & 220932 & -253319 & 15.9 & $0.0626 \pm 0.0003$ \\
$2333-327$ & 233330 & -324634 & 15.6 & $0.0518 \pm 0.0002$ \\
$2350-375$ & 235039 & -373035 & 16.5 & $0.1151 \pm 0.0008$ \\
$2353-184$ & 235321 & -182626 & 16.0 & $0.0727 \pm 0.0004$ \\
\hline \hline
\end{tabular}


Table 2. Line identifications ${ }^{1}$

\begin{tabular}{|c|c|c|c|c|c|c|c|c|c|}
\hline Object & ID & $\lambda(\AA)$ & e.w. (̊̊) & notes & Object & ID & $\lambda(\AA)$ & e.w. $(\AA)$ & notes \\
\hline \multirow[t]{5}{*}{$0005-199$} & CaII H 3933 & 4411.3 & $8.3 \pm 0.2$ & & $0940-304$ & CaII H 3933 & 4084 & $6 \pm 2.5$ & \\
\hline & CaII K 3968 & 4449.7 & $9.3 \pm 0.2$ & & & CaII K 3968 & 4120 & $6 \pm 2.5$ & \\
\hline & G-band 4304 & 4824 & $12.2 \pm 2.2$ & & & G-band 4304 & 4472.7 & $\ldots$ & \\
\hline & MgI 5175 & 5806.5 & $5.0 \pm 1.0$ & & & MgI 5175 & 5373.1 & $4.6 \pm 1.0$ & \\
\hline & Na D 5892 & 6611.6 & $5.5 \pm 0.8$ & & & $\mathrm{Ca}+\mathrm{Fe} 5269$ & 5470.7 & $1.6 \pm 0.5$ & \\
\hline \multirow[t]{5}{*}{$0013-316$} & CaII H 3933 & 4360.1 & $2.3 \pm 1.0$ & & & Na D 5892 & 6120.4 & $5.0 \pm 0.5$ & \\
\hline & CaII K 3968 & 4391.0 & $2.8 \pm 1.0$ & & $1002-320$ & CaII K & 4284.5 & $\ldots$ & \\
\hline & G-band 4304 & 4761.8 & $6.6 \pm 1.5$ & & & CaII H & 4315.8 & $\ldots$ & \\
\hline & MgI 5175 & 5727.7 & $5.1 \pm 0.8$ & & & MgI 5175 & 5634.9 & $9.4 \pm 1.0$ & \\
\hline & Na D 5892 & 6523.6 & $7.0 \pm 0.8$ & & & Na D 5892 & 6416.3 & $5.1 \pm 0.8$ & \\
\hline \multirow[t]{5}{*}{$0155-212$} & CaI H 3933 & 4562 & $\ldots$ & & $1043-290$ & CaII H & 4166.1 & $11.7 \pm 1.8$ & \\
\hline & G-band 4304 & 4988 & $1.3 \pm 0.5$ & & & CaII K & 4206.8 & $10.3 \pm 1.5$ & \\
\hline & MgI 5175 & 6005 & $6.2 \pm 1.0$ & & & G-band 4304 & 4560.8 & $7.0 \pm 1.0$ & \\
\hline & $\mathrm{Ca}+\mathrm{Fe} 5269$ & 6264 & $2.5 \pm 0.4$ & & & MgI 5175 & 5484.8 & $4.5 \pm 0.5$ & \\
\hline & NaD 5892 & 6837 & $6.8 \pm 1.0$ & & & $\mathrm{Ca}+\mathrm{Fe} 5269$ & 5585.8 & $1.4 \pm 0.4$ & \\
\hline \multirow[t]{5}{*}{$0229-208$} & CaII H 3933 & 4288.1 & $8.1 \pm 1.0$ & & & NaD 5892 & 6245.5 & $7.5 \pm 1.1$ & \\
\hline & CaII K 3968 & 4323.5 & $8.2 \pm 1.0$ & & $1257-253$ & CaII H 3933 & 4192.5 & $11 \pm 2.5$ & \\
\hline & G-band 4304 & 4684.9 & $4.0 \pm 0.5$ & & & G-band 4304 & 4584.7 & $8.5 \pm 1.4$ & \\
\hline & MgI 5175 & 5638.1 & $5.3 \pm 0.6$ & & & MgI 5175 & 5508.9 & $9.3 \pm 1.0$ & \\
\hline & Na D 5892 & 6421.7 & $5.9 \pm 0.8$ & & & $\mathrm{Ca}+\mathrm{Fe} 5269$ & 5605.7 & $\ldots$ & \\
\hline \multirow[t]{6}{*}{$0247-207$} & CaII H 3933 & 4275.2 & $\ldots$ & & & NaD 5892 & 6274.1 & $5.7 \pm 0.6$ & \\
\hline & CaII K 3968 & 4311.5 & $\ldots$ & & $1354-251$ & Ca II H & 4082.0 & $\ldots$ & \\
\hline & G-band 4304 & 4670 & $\ldots$ & & & MgI 5175 & 5370.9 & $13.0 \pm 2.0$ & \\
\hline & MgI 5175 & 5622.1 & $8.4 \pm 0.5$ & & & $\mathrm{Ca}+\mathrm{Fe} 5269$ & 5468.6 & $2.1 \pm 1.1$ & \\
\hline & $\mathrm{Ca}+\mathrm{Fe} 5269$ & 5723.8 & $1.6 \pm 0.2$ & & & Na D 5892 & 6116.4 & $3.6 \pm 0.7$ & \\
\hline & Na D 5892 & 6404.9 & $6.2 \pm 0.4$ & & $1521-300$ & MgI 5175 & 5276.9 & $13.5 \pm 2.5$ & \\
\hline \multirow[t]{4}{*}{$0257-398$} & G-band 4304 & 4591.9 & $9.5 \pm 1.5$ & & & $\mathrm{Ca}+\mathrm{Fe} 5269$ & 5369.1 & $2.2 \pm 0.9$ & \\
\hline & MgI 5175 & 5516.0 & $6.1 \pm 0.6$ & & & NaD 5892 & 6010.4 & $3.3 \pm 0.7$ & \\
\hline & $\mathrm{Ca}+\mathrm{Fe} 5269$ & 5615.7 & $\ldots$ & & & [OII] 6300 & 6423.5 & $1.9 \pm 0.8$ & $\mathrm{E}$ \\
\hline & $\mathrm{NaD} 5892$ & 6281.4 & $5.9 \pm 0.5$ & & & $\mathrm{H} \alpha$ & 6692.9 & $15.0 \pm 1.2$ & $\mathrm{E}$ \\
\hline \multirow[t]{6}{*}{$0312-343$} & CaII H 3933 & 4193.1 & $11 \pm 2$ & & & [SII] 6716 & 6848.9 & $1.5 \pm 0.3$ & $\mathrm{E}$ \\
\hline & CaII K 3968 & 4235.3 & $10.7 \pm 1.2$ & & $1929-397$ & CaII 3933 & 4225.2 & $11 \pm 3$ & \\
\hline & G-band 4304 & 4592.4 & $9.6 \pm 1.1$ & & & CaII 3968 & 4264.6 & $9.6 \pm 1.8$ & \\
\hline & MgI 5175 & 5521.9 & $13.0 \pm 2.0$ & & & G-band 4304 & 4623 & $7.8 \pm 1.7$ & \\
\hline & $\mathrm{Ca}+\mathrm{Fe} 5269$ & 5621.9 & $1.8 \pm 0.3$ & & & MgI 5175 & 5558.6 & $15.0 \pm 2.5$ & \\
\hline & NaD 5892 & 6288.5 & $7.5 \pm 0.9$ & & & Na D 5892 & 6334.3 & $8.6 \pm 0.7$ & \\
\hline \multirow[t]{7}{*}{$0344-345$} & [OIII] 5007 & 5273.4 & $5.3 \pm 1.2$ & $\mathrm{E}$ & $2209-255$ & CaII H 3933 & 4178.0 & $13 \pm 0.5$ & \\
\hline & MgI 5175 & 5449.5 & $6.4 \pm 0.4$ & & & CaII K 3968 & 4216.0 & $10 \pm 0.5$ & \\
\hline & $\mathrm{Ca}+\mathrm{Fe} 5269$ & 5551.0 & $1.9 \pm 0.4$ & & & MgI 5175 & 5501.0 & $5.5 \pm 0.5$ & \\
\hline & $\mathrm{NaD} 5892$ & 6212.1 & $7.1 \pm 0.6$ & & & $\mathrm{Ca}+\mathrm{Fe} 5269$ & 5597.2 & $2.1 \pm 0.3$ & \\
\hline & [OII] 6300 & 6637.7 & $2.7 \pm 0.4$ & & & NaD 5892 & 6263.8 & $6.2 \pm 0.5$ & \\
\hline & $\mathrm{H} \alpha$ & $\ldots$ & $>15 \pm 1.2$ & $\mathrm{~B}, \mathrm{E}$ & $2333-327$ & $\mathrm{H} \beta$ & 5113 & $2.8 \pm 0.8$ & \\
\hline & [SII] 6716-32 & $\ldots$ & $6.4 \pm 1.2$ & $\mathrm{~B}, \mathrm{E}$ & & MgI 5175 & 5444.3 & $11.4 \pm 1.5$ & \\
\hline \multirow[t]{3}{*}{$0452-190$} & MgI 5175 & 5375.6 & $4.2 \pm 0.6$ & & & $\mathrm{Ca}+\mathrm{Fe} 5270$ & 5541.0 & $2.6 \pm 0.6$ & \\
\hline & $\mathrm{Ca}+\mathrm{Fe} 5269$ & 5475.2 & $\ldots$ & & & NaD 5892 & 6198.2 & $4.5 \pm 0.5$ & \\
\hline & NaD 5892 & 6121.0 & $4.2 \pm 1.0$ & & $2350-375$ & CaII H 3933 & 4390.3 & $13.8 \pm 2.5$ & \\
\hline \multirow[t]{7}{*}{$0533-377$} & CaII K 3933 & 4316.5 & $\ldots$ & & & CaII K 3968 & 4422.5 & $12.6 \pm 2.5$ & \\
\hline & CaII H 3968 & 4347.5 & $\ldots$ & & & G-band 4304 & 4796.9 & $8.6 \pm 1.3$ & \\
\hline & G-band 4304 & 4717.7 & $6.4 \pm 1.1$ & & & MgI 5175 & 5774.8 & $10.8 \pm 1.0$ & \\
\hline & $\mathrm{H} \beta$ & 5336.8 & $2.4 \pm 0.4$ & & & $\mathrm{Ca}+\mathrm{Fe} 5270$ & 5872.3 & $2.1 \pm 0.8$ & \\
\hline & MgI 5175 & 5670.7 & $5.0 \pm 0.5$ & & & NaD 5892 & 6573.4 & $3.6 \pm 0.6$ & \\
\hline & $\mathrm{Ca}+\mathrm{Fe} 5269$ & 5775.0 & $1.6 \pm 0.4$ & & $2353-184$ & CaII 3933 & 4220.9 & $\ldots$ & \\
\hline & NaD 5892 & 6458.5 & $5.9 \pm 0.8$ & & & CaII 3968 & 4254.1 & $\ldots$ & \\
\hline \multirow[t]{4}{*}{$0712-349$} & CaII H 3933 & 4110.8 & $5 \pm 2.5$ & & & $\mathrm{H} \beta$ & 5213.9 & $2.5 \pm 1.0$ & \\
\hline & $\mathrm{Ca}+\mathrm{Fe} 5269$ & 5501.3 & $2.3 \pm 0.7$ & & & MgI 5175 & 5552.2 & $19 \pm 2.6$ & \\
\hline & Na D 5892 & 6155.8 & $5.4 \pm 0.5$ & & & Na D 5892 & 6319.7 & $6.9 \pm 1.3$ & \\
\hline & MgI 5175 & 5405.7 & $4.6 \pm 0.7$ & & & & & & \\
\hline
\end{tabular}

\footnotetext{
${ }^{1} \mathrm{E}=$ emission line; $\mathrm{B}=$ line blended with other lines or with the atmospheric absorption bands.
} 


\section{Observations and results}

Optical spectroscopy was obtained at the European Southern Observatory (ESO), La Silla, Chile, using the $1.5 \mathrm{~m}$ spectrographic telescope equipped with a Boller \& Chivens spectrograph during three observing runs in July 1993, July 1994 and February 1995. The detector employed was a Ford Aerospace CCD with $15 \mu \mathrm{m}$-pixels, which covers the range $3800-9000 \AA$ at a resolution of $3.8 \AA /$ pixel. Standard data reduction procedures were adopted to obtain 1-dimensional wavelength calibrated extracted spectra.

Spectra have also been corrected for the wavelength dependent sensitivity of the instrument.

The achieved precision on wavelength calibration was better than $\sim 1 \AA$. In all cases, a gaussian was fitted to the line profile to evaluate the line position.

In Table 1 we report for each source the measured redshift, as derived from line identifications of Table 2, together with the estimated uncertainty.

Table 3. Redshift of companions

\begin{tabular}{llrrl}
\hline \hline Radio Gal. & \multicolumn{1}{c}{$z_{\text {gal. }}$} & \multicolumn{1}{c}{$\Delta \alpha^{1}$} & \multicolumn{1}{c}{$\Delta \delta^{1}$} & \multicolumn{1}{c}{$z_{\text {comp. }}$} \\
\hline $0229-208$ & 0.0895 & 11 & -10 & $0.090 \pm 0.001$ \\
$1929-397$ & 0.0744 & -20 & 14 & $0.076 \pm 0.004$ \\
$2031-359^{2}$ & 0.088 & -9 & 24 & $0.0895 \pm 0.0003$ \\
$2333-327$ & 0.0518 & 13 & -101 & $0.0522 \pm 0.0002$ \\
\hline \hline
\end{tabular}

1 Projected distance in arcsec.

2 Coordinate of this source are (1950): 2031 32,-35 5927
Table 2 gives the measured wavelength and equivalent width of the stronger lines (Cols. 3 and 4), while Fig. 1 reports for each object the observed spectrum. All sources show a typical early-type spectrum, exhibiting the Ca II $\mathrm{H}, \mathrm{K}, G$-band, Mg I, absorption lines (Fig. 1). Only in two cases (0344-345 and 1521-300) emission lines are detected $\left(\mathrm{H}_{\alpha},[\mathrm{O} \mathrm{III}],[\mathrm{S} \mathrm{II}]\right)$. The spectrum of $0344-345$ resembles that of a Seyfert 2 .

Finally in Table 3 we give for 4 objects the redshift of a close companion galaxy, which in all cases was found to be at the same distance as the radio source. We report in Cols. 1 and 2 the name and $z$ of the radio galaxy, in Cols. 3 and 4 the projected distance in arcsec of the companion, and in the last column the measured companion's redshift.

Acknowledgements. Riccardo Scarpa wishes to thank the Visitor Program at STScI for hospitality and the "Ing. Aldo Gini" foundation for supporting this work through a fellowship. This research has made use of the NASA/IPAC Extragalactic Database (NED) which is operating by the Jet Propulsion Laboratory, Caltech, under contract with the National Aeronautics and Space Administration.

\section{References}

Ekers R.D., Wall J.V., Shaver P.A., et al. , 1989, MNRAS 236, 737

Fanaroff B.L., Riley J.M., 1974, MNRAS 167, 31

Fasano G., Falomo R., Scarpa R., 1996, MNRAS (submitted)

Pesce J.E., Falomo R., Scarpa R., 1996 (in preparation)

Tadhunter C.N., Morganti R., Di Serego Alighieri S., Fosbury

R.A.E., Danziger I.J., 1993, MNRAS 263, 999

Urry C.M., Padovani P., 1995, PASP 107, 803

Wall J.V., Peacock J.A., 1985, MNRAS 216, 173 

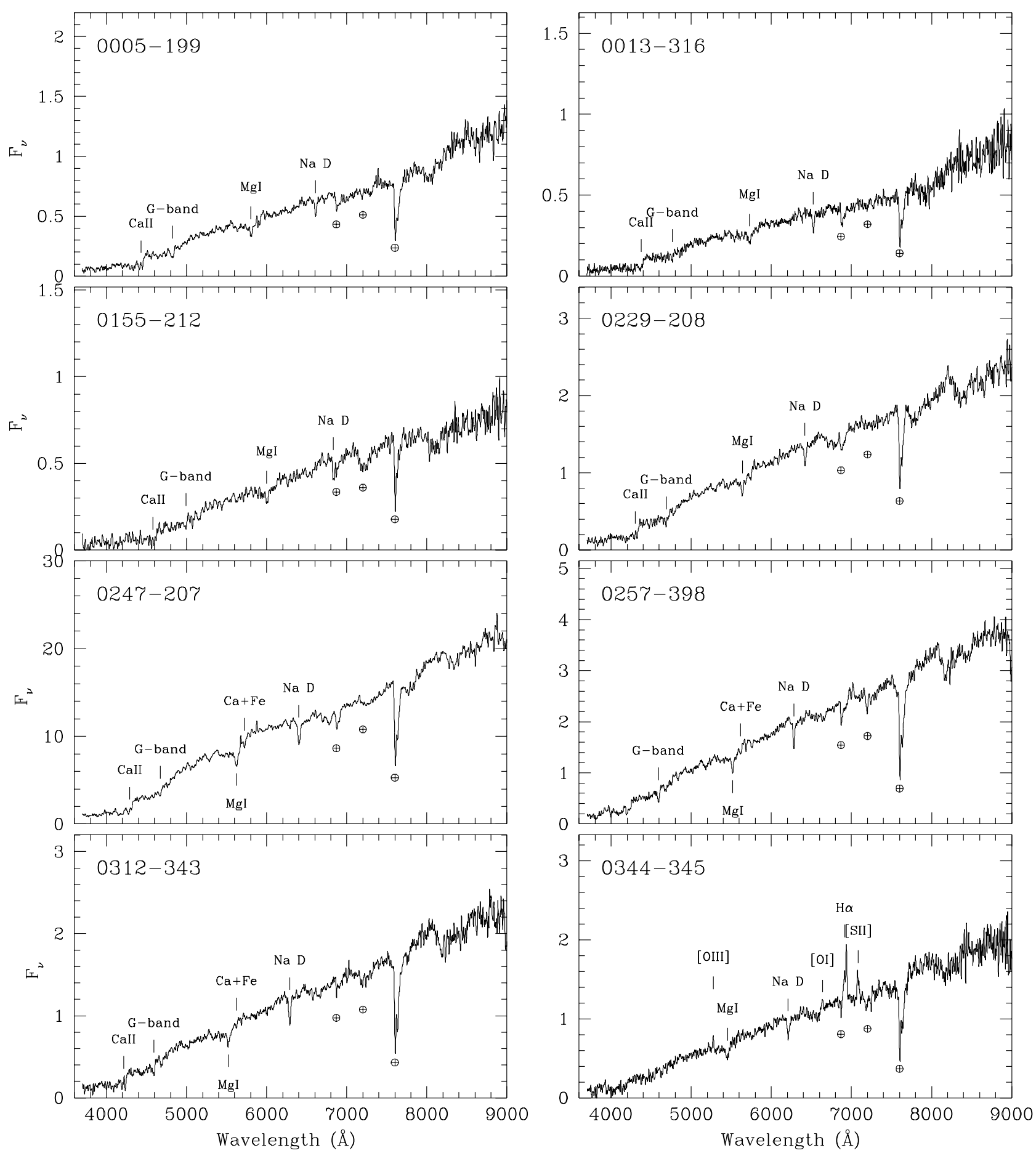

Fig. 1. Spectra of radio galaxies. Fluxes are in arbitrary units. Main features and atmospheric absorptions $(\oplus)$ are marked 

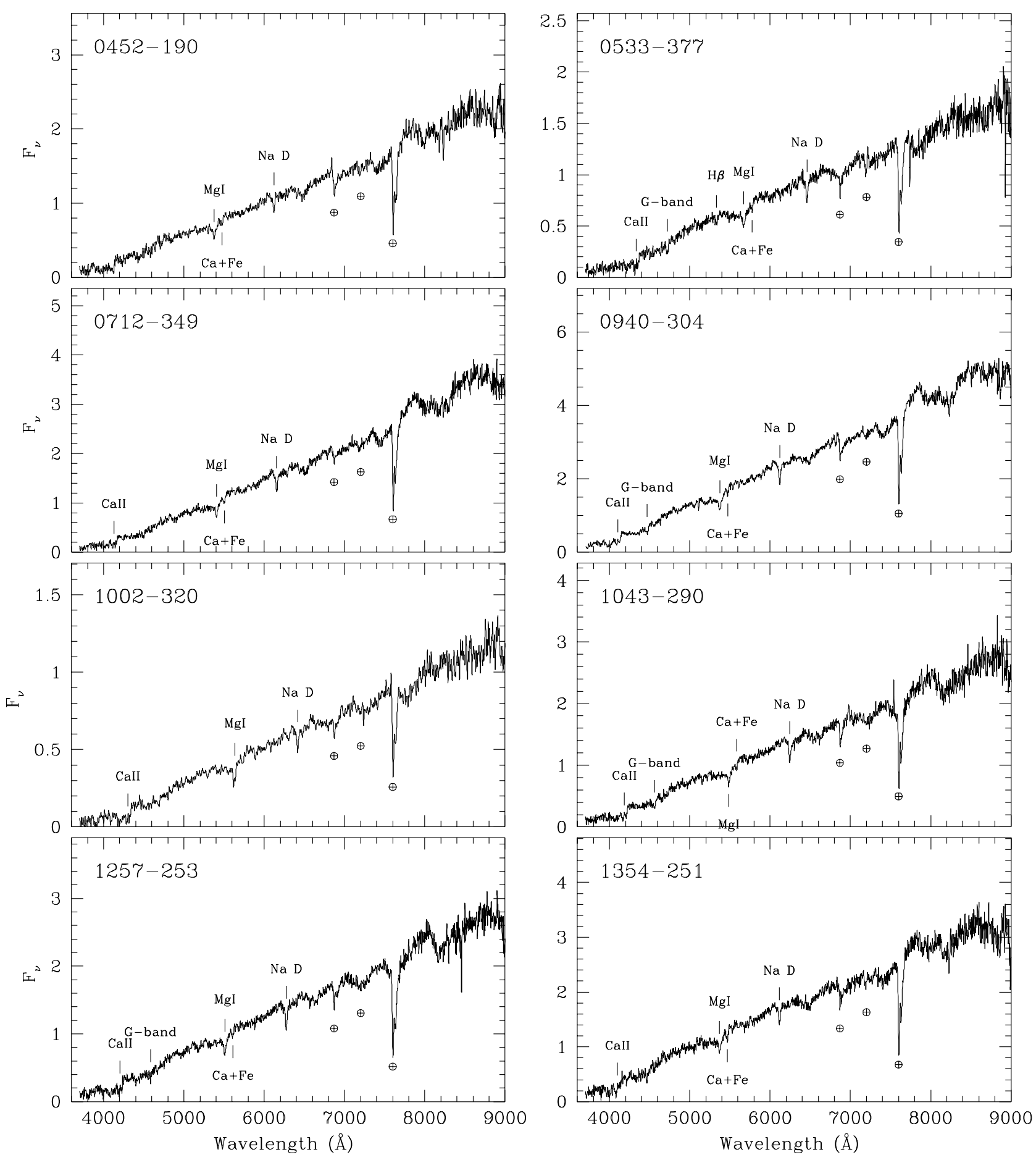

Fig. 2. 

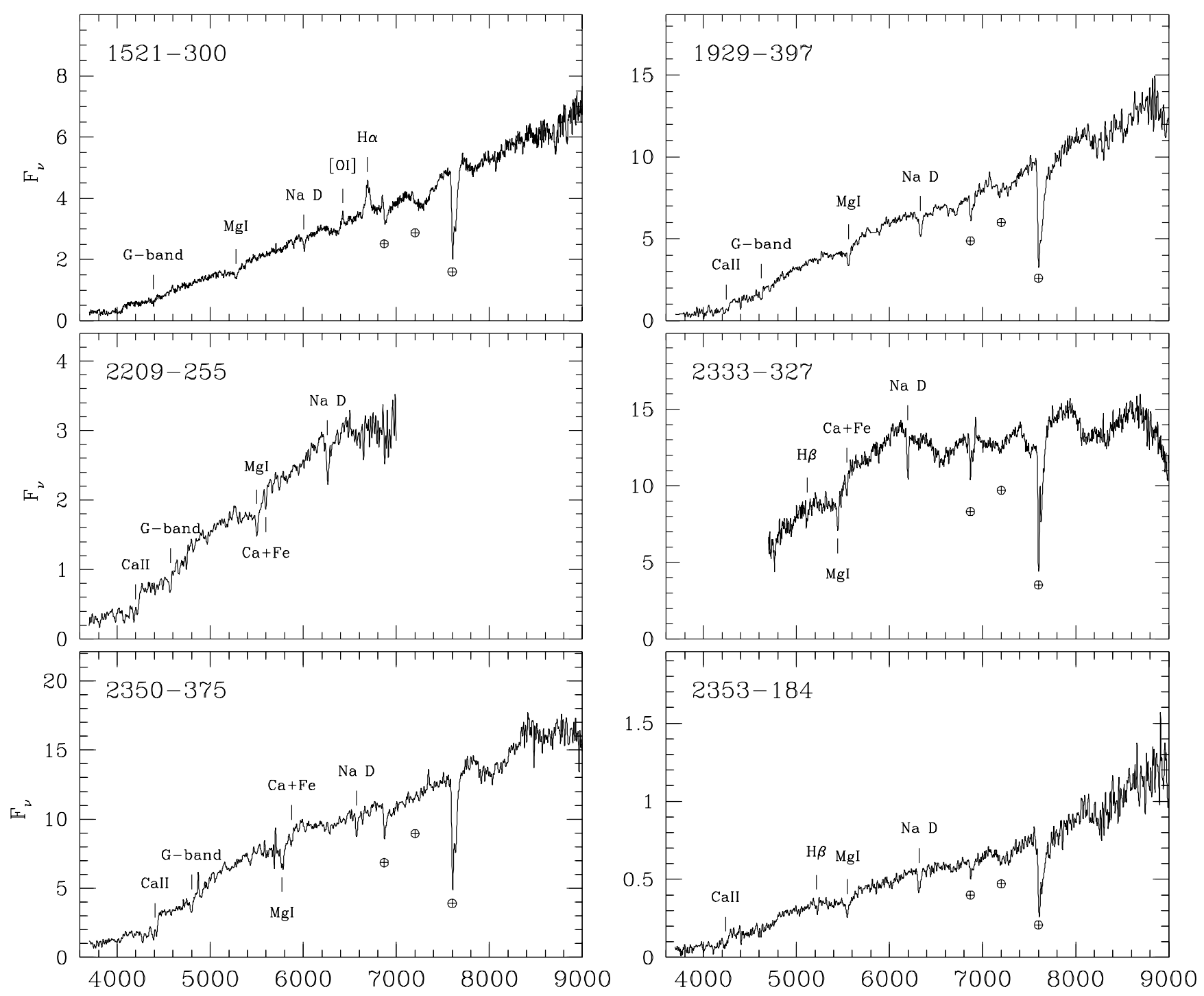

Fig. 3. 